\title{
Synthesis, Characterization and Antimicrobial Activity of Substituted Pyrazolines
}

\author{
R. KAVITHA, M NAGOOR MEERAN* and R P SURESHJEYAKUMAR
}

PG and Research Department of Chemistry, Vivekanandha College of Arts and Sciences for Women, Tiruchengode, Tamilnadu, India nagoorjmc@rediffmail.com

Received 11 August 2015 / Accepted 2 September 2015

\begin{abstract}
Some novel series of substituted pyrazoline were synthesized from chalcones. Various substituted pyrazoline prepared by cyclization of chalcones with substituted phenylhydrazine in ethanolic solution. The synthesized compounds were characterized by their physical properties of $\mathrm{IR},{ }^{1} \mathrm{H} \&{ }^{13} \mathrm{C}$ NMR and elemental analysis studies. The antimicrobial activity of all the compounds (CP01-CP04) showed significant activity against all the bacteria and fungus.
\end{abstract}

Keywords: Chalcones, Pyrazoline, Phenylhydrazine, Antimicrobial activity

\section{Introduction}

Pyrazoles are five member ring heterocyclic compounds, have some structural features with two nitrogen atoms in adjacent position and are also called as azoles ${ }^{1}$. The best described property of almost every group of pyrazoles is in the treatment of inflammation and inflammation associated discord, such as arthritis ${ }^{2}$. Pyrazole derivatives are the subject many research studies due to their widespread potential biological activities such as antimicrobial ${ }^{3}$, antiviral $^{4}$, antitumor ${ }^{5,6}$, antihistaminic ${ }^{7}$, antidepressant ${ }^{8}$, insecticides and fungicides ${ }^{9}$.

Several pyrazole derivatives have been found to possess significant activities such as 5 $\alpha$-red-ukase inhibitor ${ }^{10}$, anttiproliferative ${ }^{11}$, antiphrastic ${ }^{12}$, herbicides ${ }^{13}$, a good number of pyrazoles have also been reported to have interesting biological activities like antiinflammatory $^{14}$ and antiprotozoal ${ }^{15-16}$ which render them valuable active ingredients of medicine and plant protecting agents. Further current literature indicates 1,2 pyrazole derivatives to possess various biological activities ${ }^{17}$.

These compounds are useful in the field of medicine and are used as a starting material for the synthesis of new drugs ${ }^{18-27}$. In view of these data we have undertaken the synthesis, characterization and antimicrobial evaluation of substituted pyrazolines. All the synthesized compounds were characterized on the basis of their physical properties, IR, ${ }^{1} \mathrm{H} \&{ }^{13} \mathrm{C} \mathrm{NMR}$ spectral data and elemental analysis. The physical data of titled compounds are summarized in Table 1 . 
Table 1. Physical and analytical data of the synthesized compounds

\begin{tabular}{|c|c|c|c|c|c|c|c|c|c|c|}
\hline \multirow[t]{2}{*}{ Compd. } & \multirow[t]{2}{*}{$\mathrm{R}$} & \multirow[t]{2}{*}{$\mathrm{R}^{\prime}$} & \multirow[t]{2}{*}{ Compound Name } & \multirow{2}{*}{$\begin{array}{l}\text { Molecular } \\
\text { formula }\end{array}$} & \multirow{2}{*}{$\begin{array}{l}\text { M. } \\
\text { Wt }\end{array}$} & \multirow{2}{*}{$\stackrel{\mathrm{M} . P}{{ }^{\mathrm{O}} \mathrm{C}}$} & \multirow{2}{*}{$\begin{array}{l}\% \\
\text { Yield }\end{array}$} & \multicolumn{3}{|c|}{$\begin{array}{l}\text { Elemental Analysis \% } \\
\text { (Calculated) Found }\end{array}$} \\
\hline & & & & & & & & $\mathrm{C}$ & $\mathrm{H}$ & $\mathrm{N}$ \\
\hline $\mathrm{CO} 1$ & $3-\mathrm{NO}_{2}$ & - & 1-(3-nitrophenyl)-3-phenylprop-2-en- & $\mathrm{C}_{15} \mathrm{H}_{11} \mathrm{NO}_{2}$ & 253.25 & 204 & 56 & $(71.07)$ & $(4.34)$ & $(5.52)$ \\
\hline & & & $\begin{array}{c}\text { 1-one } \\
13 \text { ampions }\end{array}$ & & & & & 71.05 & 4.32 & 5.50 \\
\hline $\mathrm{CO} 3$ & $3-\mathrm{NH}_{2}$ & - & $\begin{array}{c}\text { 1-(3-amniophenyl)-3-phenyl prop-2- } \\
\text { en-1-one }\end{array}$ & $\mathrm{C}_{15} \mathrm{H}_{13} \mathrm{NO}$ & 223.15 & 198 & 62 & $\begin{array}{c}(80.66) \\
80.85\end{array}$ & $\begin{array}{c}(5.82) \\
5.85\end{array}$ & $\begin{array}{c}(6.27) \\
6.25\end{array}$ \\
\hline CPO1 & $3-\mathrm{NH}_{2}$ & $\mathrm{H}$ & 3-(1,5 diphenyl-4,5-drohydro-1,4 & $\mathrm{C}_{15} \mathrm{H}_{19} \mathrm{~N}_{3}$ & 313.39 & 223 & 60 & $(80.41)$ & $(6.06)$ & $(13.40)$ \\
\hline - & ग & & & $601019+3$ & & & & 80.40 & 6.05 & 13.42 \\
\hline $\mathrm{CPO} 2$ & $3-\mathrm{NH}_{2}$ & 2,4-di- & 3 [1(2,4 dinitro phenyl)-5-phenyl-4,5 & $\mathrm{C}_{21} \mathrm{H}_{17} \mathrm{~N}_{5} \mathrm{O}_{4}$ & 403.39 & 217 & 64 & $(62.47)$ & $(4.21)$ & $(17.35)$ \\
\hline & & $\mathrm{NO}_{2}$ & dihydro)-1- pyrazol-3-yl)aniline & & & & & $\begin{array}{c}62.45 \\
(73.39)\end{array}$ & $\begin{array}{c}4.20 \\
(4.95)\end{array}$ & $\begin{array}{l}17.32 \\
(1223)\end{array}$ \\
\hline CPO3 & $3-\mathrm{NO}_{2}$ & $\mathrm{H}$ & dihydro-1-pyrazole & $\mathrm{C}_{21} \mathrm{H}_{17} \mathrm{~N}_{3} \mathrm{O}_{2}$ & 343.37 & 203 & 59 & 73.40 & 4.98 & 12.25 \\
\hline CPO4 & $3-\mathrm{NO}_{2}$ & $\begin{array}{c}2,4-\mathrm{di}- \\
\mathrm{NO}_{2}\end{array}$ & $\begin{array}{l}\text { [1(2,4 dinitro phenyl)-3-(3- dinitro } \\
\text { phenyl) -5-phenyl-4,5-dihydro)-1- } \\
\text { pyrazole }\end{array}$ & $\mathrm{C}_{21} \mathrm{H}_{15} \mathrm{~N}_{5} \mathrm{O}_{6}$ & 433.75 & 214 & 62 & $\begin{array}{c}(58.09) \\
58.10\end{array}$ & $\begin{array}{c}(3.45) \\
3.40\end{array}$ & $\begin{array}{c}(16.13) \\
16.10\end{array}$ \\
\hline
\end{tabular}




\section{Experimental}

The melting points were carried out in open capillary tube and were uncorrected. Thin layer chromatography was performed using silica gel coated on a glass plate and spots were visualized by exposure to iodine vapor. IR spectra of compounds were scanned on Shimadzu IR spectrophotometer using $\mathrm{KBr}$ disc and expressed in $\mathrm{cm}^{-1} .{ }^{1} \mathrm{H} \&{ }^{13} \mathrm{C}$ NMR spectra were recorded in DMSO-D ${ }_{6}$ on BRUKER $(400 \mathrm{MHz})$ spectrometer using TMS as an internal standard (chemical shifts in $\delta, \mathrm{ppm}$ ) (Table 2). The elemental analysis for $\mathrm{C}, \mathrm{H}$, and $\mathrm{N}$ were in an agreement with the calculated values. The synthesis of the target compounds was accomplished according to the reaction sequence illustrated in Scheme 1.

Table 2. Spectral data of the compounds

\begin{tabular}{|c|c|c|c|}
\hline Compd. & $\mathrm{IR}(\mathrm{KBr}) v_{\max }$ in $\mathrm{cm}^{-1}$ & $\begin{array}{c}\left.{ }^{1} \mathrm{H} \text { NMR(DMSO-d }{ }_{6}\right), \delta, \\
\text { ppm }\end{array}$ & $\begin{array}{c}{ }^{13} \mathrm{C} \text { NMR-DMSO- } \\
\left.\mathrm{d}_{6}\right), \delta, \mathrm{ppm}\end{array}$ \\
\hline $\mathrm{C} 01$ & $\begin{array}{c}3086 \text { (aromatic } \mathrm{C}-\mathrm{H} \text { str), } 2624 \\
\text { (aliphatic } \mathrm{C}-\mathrm{H} \text { str), } 1658 \\
(\mathrm{C}=\mathrm{O}), 1604 \text { (aromatic } \mathrm{C}=\mathrm{C}) \\
1442 \text { (aliphatic } \mathrm{C}=\mathrm{C} \text { str), } 1342 \\
\left(\mathrm{NO}_{2} \text { str). }\right.\end{array}$ & $\begin{array}{l}\text { 4.587(s, } 1 \mathrm{H} \text {, aliphatic C-H }) \\
4.331(\mathrm{~s}, 1 \mathrm{H} \text {, aliphatic C-H }) \\
7.482-8.828(\mathrm{~m}, 9 \mathrm{H}, \mathrm{Ar}-\mathrm{H})\end{array}$ & $\begin{array}{c}187(\mathrm{C}=\mathrm{O}), 121- \\
148(\mathrm{Ar}-\mathrm{H}, \& \\
\mathrm{HC}=\mathrm{CH})\end{array}$ \\
\hline $\mathrm{C} 03$ & $\begin{array}{c}3464 \text { (NH str), } 3055 \\
\text { (aromatic C-H str), } 2924 \\
\text { (aliphatic C-H str), } 1658 \\
\text { (C=O str), } 1589 \text { (aromatic } \\
\mathrm{C}=\mathrm{C} \text { str), } 1489 \text { (aliphatic } \\
\mathrm{C}=\mathrm{C} \text { str). }\end{array}$ & $\begin{array}{c}2.72\left(\mathrm{~s}, 2 \mathrm{H}, \mathrm{NH}_{2}\right) 4.17 \\
(\mathrm{~s}, 1 \mathrm{H}, \text { aliphatic } \mathrm{C}-\mathrm{H}), 4.25 \\
(\mathrm{~s}, 1 \mathrm{H}, \text { aliphatic } \mathrm{C}-\mathrm{H}), \\
\text { 6.89-7.94 (m, 9H, Ar-H). }\end{array}$ & $\begin{array}{c}196(\mathrm{C}=\mathrm{O}), 117- \\
150(\mathrm{Ar}-\mathrm{H}, \& \\
\mathrm{HC}=\mathrm{CH})\end{array}$ \\
\hline CP01 & $\begin{array}{c}\text { 3309(NH str), } 3032 \text { (aromatic } \\
\text { C-H str), } 2924 \text { (aliphatic C-H } \\
\text { str), } 1666 \text { (C=N str), } 1597 \\
\text { (aromatic C=C), } 1489 \\
\text { (aliphatic C-C str). }\end{array}$ & $\begin{array}{c}2.063(\mathrm{~s}, 2 \mathrm{H}, \text { Ar-NH}), \\
\text { pyrazoline ring, 3.011-3.070 } \\
\left(\mathrm{dd}, 1 \mathrm{H}, \mathrm{H}_{\mathrm{A}}\right), 3.814-3.888 \\
\left(\mathrm{dd}, 1 \mathrm{H}, \mathrm{H}_{\mathrm{M}}\right), 5.161(\mathrm{dd}, 1 \mathrm{H}, \\
\left.\mathrm{H}_{\mathrm{X}}\right), 6.702-8.047(\mathrm{~m}, 9 \mathrm{H}, \\
\text { Ar-H); }\end{array}$ & $\begin{array}{c}168(\mathrm{C}=\mathrm{N}), 111- \\
147 \quad(\mathrm{Ar}-\mathrm{C}), \\
\text { pyrazoline ring, } 63 \\
\left(\mathrm{CH}_{2}\right), 23(\mathrm{CH})\end{array}$ \\
\hline CP02 & $\begin{array}{c}3456 \text { (NH str), } 3086 \\
\text { (aromatic C-H str), } 2924 \\
\text { (aliphatic C-H str), } 1666 \\
(\mathrm{C}=\mathrm{N} \text { str), } 1604 \text { (aromatic } \\
\mathrm{C}=\mathrm{C}), 1419 \text { (aliphatic C-C } \\
\text { str), and } 1327\left(\mathrm{NO}_{2} \text { str). }\right. \\
\end{array}$ & $\begin{array}{c}2.075(\mathrm{~s}, 2 \mathrm{H}, \mathrm{Ar}-\mathrm{NH}), \\
\text { pyrazoline ring, } 3.22(\mathrm{dd}, \\
\left.1 \mathrm{H}, \mathrm{H}_{\mathrm{A}}\right), 4.010\left(\mathrm{dd}, 1 \mathrm{H}, \mathrm{H}_{\mathrm{M}}\right), \\
5.582\left(\mathrm{dd}, 1 \mathrm{H}, \mathrm{H}_{\mathrm{X}}\right), 7.116- \\
8.868(\mathrm{~m}, 9 \mathrm{H}, \mathrm{Ar}-\mathrm{H})\end{array}$ & $\begin{array}{c}169(\mathrm{C}=\mathrm{N}), 116- \\
149(\mathrm{Ar}-\mathrm{C}), \\
\text { pyrazoline ring, } 63 \\
\left(\mathrm{CH}_{2}\right), 23(\mathrm{CH})\end{array}$ \\
\hline СР03 & $\begin{array}{c}3032 \text { (aromatic C-H str), } \\
2924 \text { (aliphatic C-H str), } \\
1674 \text { (C=N str), } 1597 \\
\text { (aromatic C=C str), } 1496 \\
\text { (aliphatic C-C str), and } 1350 \\
\left(\mathrm{NO}_{2} \text { str) }\right.\end{array}$ & $\begin{array}{c}\text { pyrazoline ring, 3.169-3.227 } \\
\left(\mathrm{dd}, 1 \mathrm{H}, \mathrm{H}_{\mathrm{A}}\right), 3.938- \\
4.013\left(\mathrm{dd}, 1 \mathrm{H}, \mathrm{H}_{\mathrm{M}}\right), 5.555- \\
5.600\left(\mathrm{dd}, 1 \mathrm{H}, \mathrm{H}_{\mathrm{X}}\right), 6.743-- \\
8.476(\mathrm{~m}, 14 \mathrm{H}, \mathrm{Ar}-\mathrm{H})\end{array}$ & $\begin{array}{l}163(\mathrm{C}=\mathrm{N}), 105- \\
148 \\
(\mathrm{Ar}-\mathrm{C}), \text { pyrazoline } \\
\text { ring, } \\
\left(\mathrm{CH}_{2}\right), 22(\mathrm{CH}) .\end{array}$ \\
\hline P04 & $\begin{array}{c}3032 \text { (aromatic C-H str), } 2924 \\
\text { (aliphatic C-H str), } 1674(\mathrm{C}=\mathrm{N} \\
\text { str), } 1597 \text { (aromatic C=C str), } \\
1496 \text { (aliphatic C-C str), and } \\
1350\left(\mathrm{NO}_{2} \text { str) }\right.\end{array}$ & $\begin{array}{c}\text { pyrazoline ring, 3.169-3.227 } \\
\left(\mathrm{dd}, 1 \mathrm{H}, \mathrm{H}_{\mathrm{A}}\right), 3.938-4.013(\mathrm{dd} \\
\left.1 \mathrm{H}, \mathrm{H}_{\mathrm{M}}\right), 5.555-5.600(\mathrm{dd}, 1 \mathrm{H} \\
\left.\mathrm{H}_{\mathrm{X}}\right), 6.743--8.476(\mathrm{~m}, 14 \mathrm{H}, \\
\text { Ar-H); }\end{array}$ & $\begin{array}{c}163(\mathrm{C}=\mathrm{N}), 105- \\
148(\text { Ar-C }), \\
\text { pyrazoline ring, } 63 \\
\left(\mathrm{CH}_{2}\right), 22(\mathrm{CH}) .\end{array}$ \\
\hline
\end{tabular}


<smiles>[R]c1cccc(C(C)=O)c1</smiles><smiles>[R][X]c1ccccc1C(=O)/C=C/c1ccccc1</smiles>

$\mathrm{C} 01-\mathrm{C} 03$

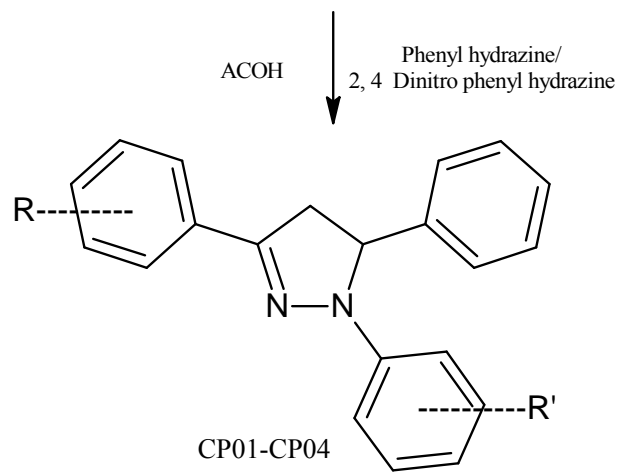

$\mathrm{C} 01: \mathrm{R}=3 \mathrm{NO}_{2} \mathrm{C} 03: \mathrm{R}=3 \mathrm{NH}_{2} ; \mathrm{CP01}: \mathrm{R}=3 \mathrm{NH}_{2}, \mathrm{R}^{\prime}=\mathrm{H} ; \mathrm{CP} 02: \mathrm{R}=3 \mathrm{NH}_{2}, \mathrm{R}^{\prime}=2,4-$ di- $\mathrm{NO}_{2} ; \mathrm{CP} 03$ : $\mathrm{R}=3 \mathrm{NO}_{2}, \mathrm{R}^{\prime}=\mathrm{H} ; \mathrm{CP04}: \mathrm{R}=3 \mathrm{NO}_{2}, \mathrm{R}^{\prime}=2,4-\mathrm{di}-\mathrm{NO}_{2}$

\section{General procedure}

\section{Scheme 1}

Synthesis of 1-(3-nitrophenyl)-3-phenylprop-2-en-1-one $(\mathrm{CO1})^{28}$

A mixture of 3-nitro acetophenone $(0.01 \mathrm{~mol})$ and benzaldehyde $(0.01 \mathrm{~mol})$ was dissolved in ethanolic $\mathrm{NaOH}(20 \mathrm{~mL})$ and stirred about 2-3 hours with a mechanical stirrer and kept in refrigerator for 24 hours. The content was poured into crushed ice and acidified with $\mathrm{HCl}$. The solid separated was filtered and recrystalization from ethanol.

\section{Synthesis of 1-(3-amniophenyl)-3-phenyl prop-2-en-1-one (C03)}

A mixture of 3-amnio acetophenone (I) $(0.05 \mathrm{~mol})$ and benzaldehyde $(0.05 \mathrm{~mol})$ was dissolved in ethanolic $\mathrm{NaOH}(20 \mathrm{~mL})$ and stirred about 2-3 hours with a mechanical stirrer and kept in refrigerator for 24 hours. The content was poured into crushed ice and acidified with $\mathrm{HCl}$. The solid separated was filtered and recrystalisation from ethanol.

\section{Synthesis of substituted pyrazolines ${ }^{29}$ (CP01-CP04)}

A mixture of chalcones ( 0.001 mole) and appropriate phenylhydrazine and substituted phenyl hydrazine $(0.001$ mole $)$ in glacial acid $(25 \mathrm{~mL})$ was heated under refluxed for 4 hours, then the mixture was poured in to ice water $(100 \mathrm{~mL}$. The precipitate obtained was filtered washed with water and recrystallized from absolute ethanol.

\section{Antimicrobial activity}

In vitro antibacterial activity was determined by Kirby-Bauer disc diffusion method against bacteria such as Staphylococcus aureus and Bacillus (Gram +ve), Salmonella typhi and Pseudomonas aeruginosa (Gram -ve) using Ampicilin as standard a standard drug. The standard and test compounds were prepared in DMSO at different concentrations. The zone of inhibition was compared with standard drug after $24 \mathrm{~h}$ incubation at $35-37{ }^{\circ} \mathrm{C}$. 
Similarly antifungal activity was performed against Candida. The standard and test compounds were prepared in DMSO at different concentrations. The zone of inhibition was compared with standard drug after $48 \mathrm{~h}$ at $25{ }^{\circ} \mathrm{C}$. The results of antibacterial and antifungal activity are presented in Table 3.

\section{Results and Discussion}

Synthesis of substituted pyrazolines was obtained through above Scheme 1. The required chalcones $(\mathrm{C} 01, \mathrm{C} 03)$ was synthesized according to the procedure reported in the literature. Chalcones with phenylhydrazine gave good yield of pyrazolines (CP01-CP04). The spectral analysis of all the compounds was done by $\mathrm{IR},{ }^{1} \mathrm{H}$ and ${ }^{13} \mathrm{C}$ NMR and the spectral data were consistent with the assigned structures.

In vitro antibacterial activity of selected compounds was carried out by Kirby-Bauer disc diffusion method against bacteria such as Staphylococcus aureus, Bacillus, Salmonella typhi, Pseudomonas aeruginosa. The compounds CP01-CP04 showed significant activity against all the bacteria. Antifungal activity was performed on candida. The compounds $\mathrm{CP} 01, \mathrm{CP} 03$, CP04 showed moderate activity against the fungus. The compound $\mathrm{CP} 02$ was more active among screened compounds.

Table 3. Antimicrobial activity of the synthesized compounds Zone of inhibition ( $\mathrm{mm}$ ) of synthesized compounds

\begin{tabular}{|c|c|c|c|c|c|c|c|c|c|c|c|c|c|c|c|c|c|c|c|c|}
\hline \multirow{4}{*}{ 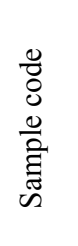 } & \multicolumn{16}{|c|}{ Anti-bacterial activity } & \multirow{2}{*}{\multicolumn{4}{|c|}{$\begin{array}{l}\text { Anti-fugal } \\
\text { activity }\end{array}$}} \\
\hline & \multicolumn{8}{|c|}{ Gram positive } & \multicolumn{8}{|c|}{ Gram negative } & & & & \\
\hline & \multicolumn{4}{|c|}{ Staphylococcus.spp } & \multicolumn{4}{|c|}{ Bacillus.spp } & \multicolumn{4}{|c|}{ Salmonella.spp } & \multicolumn{4}{|c|}{ Pseudomonas.spp } & \multicolumn{4}{|c|}{ Candida } \\
\hline & $\begin{array}{l}\infty 0 \\
\stackrel{0}{g} \\
8 \\
\Xi\end{array}$ & 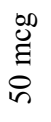 & $\begin{array}{l}\text { DD } \\
\stackrel{\Xi}{\Xi} \\
\beth\end{array}$ & 吾 & $\begin{array}{l}00 \\
\stackrel{\Xi}{\Xi} \\
8\end{array}$ & $\begin{array}{l}\text { of } \\
\text { : } \\
\text { in } \\
\text { in }\end{array}$ & 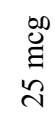 & $\vec{D}$ & $\begin{array}{l}000 \\
\ddot{\Xi} \\
8 \\
\Xi\end{array}$ & $\begin{array}{l}0 \\
\stackrel{0}{0} \\
\stackrel{0}{n}\end{array}$ & $\begin{array}{l}\text { on } \\
\ddot{\Xi} \\
\end{array}$ & 吾 & $\begin{array}{l}\stackrel{0}{0} \\
\stackrel{\Xi}{8} \\
\stackrel{0}{0}\end{array}$ & $\begin{array}{l}\text { 足 } \\
\stackrel{g}{\Xi} \\
\text { in }\end{array}$ & 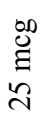 & i & $\begin{array}{l}00 \\
\stackrel{0}{\Xi} \\
8\end{array}$ & $\begin{array}{l}\text { on } \\
\stackrel{\Xi}{\Xi} \\
\stackrel{8}{n}\end{array}$ & $\begin{array}{l}\infty \\
\stackrel{0}{\Xi} \\
\tilde{a}\end{array}$ & 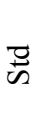 \\
\hline $\mathrm{CP}$ & 7 & 5 & 4 & 11 & 7 & 3 & - & 11 & 3 & 2 & - & 9 & 6 & 4 & 3 & 9 & 8 & - & - & \\
\hline СР02 & 6 & 5 & 2 & 5 & 8 & 6 & 5 & 6 & 5 & 3 & - & 5 & 8 & 7 & 5 & 10 & 9 & 6 & 4 & 12 \\
\hline СР03 & 8 & 6 & 4 & 9 & 9 & 6 & 5 & 9 & 9 & 8 & 5 & 10 & 9 & 8 & 6 & 8 & 8 & 5 & 3 & 21 \\
\hline СР04 & 9 & 7 & 6 & 12 & 13 & 9 & 5 & 10 & 6 & 5 & 3 & 10 & 9 & 8 & 5 & 10 & 7 & 5 & 4 & 15 \\
\hline
\end{tabular}

\section{Conclusion}

In conclusion, the reaction profile explained in the present work is very efficient to synthesized substituted pyrazolines. The prepared compounds showed potent antimicrobial activities and these are promising compounds for further pharmacological studies.

\section{References}

1. Eicher T and Hauptmann S, 2003 Edition Ind. The Chemistry of Heterocyclic: Structure, Reactions, Syntheses and Applications, Wiley- VCH, ISBN3527307206.

2. John J, Tally Donald J and Rogier Jr, Both of St, Louis, Mo G D. Searle \&.Co,. Skokie, 1995 Pt No: 5, 434, 178.

3. Pimerova E V and Voronina E V, Pharm Chem J., 2001, 35, 18-20.

4. Janus S L, Magdif A Z, Erik B P and Claus N, Monatshefte für Chem., 1999, 130(9), 1167-1174; DOI:10.1007/PL00010295

5. Park H J, Lee K, Park S, Ahn B, Lee J C, Cho H Y and Lee K I. Bioorg Med Chem Lett., 2005, 15(13), 3307-3312; DOI:10.1016/j.bmcl.2005.03.082

6. Bouabdallah I, M'barek L A,. Zyad A, Ramadan A, Zidane I and Melhaoui A, Nat Prod Res., 2006, 20(11), 1024-1030; DOI:10.1080/14786410600921441 
7. Yildirim I, Ozdemir N, Akçamur Y, Dinçer M and Andaç O, Acta Cryst., 2005, E61, 256-258.

8. Bailey D M, Hansen P E, Hlavac A G, Baizman E R, Pearl J, Defelice A F, Feigenson M E, J Med Chem., 1985, 28(2), 256-260; DOI:10.1021/jm00380a020

9. Chu C K and Cutler J, J Heterocycl Chem., 1986, 23(2), 289-319; DOI:10.1002/jhet.5570230201

10. Amr AEI G E S, Abdel-Latif N A and Abdlla M M, Acta Pharm., 2006, 56, 1203.

11. Chimichi S, Boccalini M, Hassan M M M, Viola G, Dall' Acqua F and Curini M, Tetrahedron, 2006, 62(1), 90-96; DOI:10.1016/j.tet.2005.09.135

12. Henry T A, The Plant Alkoloids, Anmol Publicaion Pvt. Ltd,. 1999.

13. Kobayashi, Hisafumi, Kato Motto, Nitani, Fumio, Chem Abstr., 1989, 106 297008/g

14. Nugent R A, Marphy M, Stephen T S, Colin J D, Robert J S, Nigel D S, Louise A G, Sharon K S, Danielle G A, Karen A R and Norman A R, J Med Chem., 1993, 36(1), 134-139; DOI:10.1021/jm00053a017

15. Hantoon M A, Minnesota Medicine, 2001, 84, 102.

16. Zhang X, Li X, Allan G F and Sbriscia T, J Med Chem., 2007, 50(16), 3857-3869; DOI:10.1021/jm0613976

17. Abunada N M, Hasaneen H M, Kandile N G and Miqdad O A, Molecules, 2008, 13(7), 1501-1517; DOI:10.3390/molecules13071501

18. Smith I K, Time, 2000, 155(16), 89.

19. Mao Y and Mao X, Faming Zhu Shenq Gong Shuom., 2003, 23, 468-470.

20. Tang X, Serizawa A, Tokunaga M, Yasuda M, Matsushita K, Terachi T and Osamura R, Human Patho., 2006, 37, 1187-1192.

21. Mohsin M, J Med Sci., 2008, 1(1), 42-49.

22. Kato M, Ayumi, 2009, 230, 458-462.

23. Priddy D B, Franks M, Konas M, Vrana M A, Yoon T H and McGrath J E, Polym Preprints, 1993, 34, 310-311.

24. Khananashvili L, Markarashvili E, Vardosanidze V, Tkeshelashvili R, Butskhrikidze B, Nogaideli G and Tsomaia N, Izv Akad Nauk Gruzii, 2001, 27, 48-52.

25. Guan W, Li X and Xiao S, Zhong Yao Yu Linch., 2005, 5, 283-285.

26. Ackermann L, Althammer A and Mayer P, Synthesis, 2009, 20, 3493-3503; DOI:10.1055/s-0029-1216977

27. Sammour A E A, Tetrahedron, 1967, 20(4), 1067-1071; DOI:10.1016/S00404020(01)98444-3

28. Vishal D Joshi, Mahendra D Kshirsgar and Sarita Singhal, Int J Chem Tech Res., 2012, 4(3), 971-975

29. Ahmed Abdul Hassan AI-Kadhimi, Ahmed K AI-Khayate, Mohammed G ALDulyme, The F Scientific Conference the collage of Sciences, 2013. 